Pacific Journal of Mathematic 


\title{
AN OPERATIONAL CALCULUS FOR OPERATORS WITH SPECTRUM IN A STRIP
}

\author{
WILLIAM G. BADE
}

1. Introduction. Let $X$ be a complex Banach space, and $T$ be a closed distributive operator whose domain and range are in $X$. We suppose the spectrum $\sigma(T)$ of 7 does not cover the whole plane, and write

$$
(\lambda I-T)^{-1}=R_{\lambda}(T)
$$

for $\lambda \notin \sigma(T)$. In the case that $T$ is bounded, N. Dunford [2] and A. E. Taylor [13] have defined an operational calculus for $T$ by the formula

$$
f(T)=\frac{1}{2 \pi i} \int_{C} f(\lambda) R_{\lambda}(T) d \lambda,
$$

where $f$ is analytic on $\sigma(T)$, and $C$ is a suitable bounded contour enclosing $\sigma(T)$. Such functions $f$ form an algebra, and the mapping $f \longrightarrow f(T)$ is a homomorphism of this algebra into the algebra of bounded operators on $X$.

When $T$ is assumed to be closed but not bounded, the problem of developing an operational calculus for $T$ meets with the difficulties that the domain $D(T)$ is a proper subspace, and $\sigma(T)$ is in general unbounded. A modification of ( 1.1 ),

$$
f(T)=f(\infty) I+\frac{1}{2 \pi i} \int_{C} f(\lambda) R_{\lambda}(T) d \lambda,
$$

has been used by Taylor [14] when $f$ is analytic on $\sigma(T)$ and at infinity. Here $C$ is a bounded contour enclosing the singularities of $f$. Although most of the theory for the bounded case may be carried over, the class of functions $f$ is restricted; and polynomials in $T$, being unbounded operators, need a separate

Received June 13, 1952. This paper is a revised version of the author's doctoral dissertation submitted to the University of California, Los Angeles. He wishes to thank Professor Angus E. Taylor for constant help and encouragement.

Pacific J. Math. 3 (1953), 257-290 
treatment.

In this paper we consider the case that $\sigma(I)$ lies in a strip $S$ of finite width, and $\left\|R_{\lambda}(T)\right\|$ is bounded outside any strip containing $S$ in its interior. This is a common situation for differential operators (for example, $T=d / d t$ in $\left.L_{p}(-\propto, \propto), p \geq 1\right)$. These assumptions enable us to define an operator corresponding to any function analytic and of finite order in a strip containing $S$. The operator $f(T)$ is bounded or unbounded depending on the growth behavior of $f$. In this it resembles the operational calculus for unbounded self-adjoint operators in llilbert space $([8], \mid 12])$, and in fact reduces to it in this case.

In $2-4$ the calculus is constructed from a postulated set of conditions on $I$ and $K_{\lambda}(T)$. If $f$ is absolutely integrable in a strip containing $S$, the operator $f(T)$ is defined by a variant of formula (1.1),

$$
f(T)=\frac{1}{2 \pi i} \int_{\Gamma} f(\lambda) R_{\lambda}(T) d \lambda
$$

where $\Gamma$ is an infinite contour running up one side of $S$ and down the other. If $f$ is of order $n-2$, roughly speaking, then $f(T) x$ is defined for $x$ in the subspace

$$
l_{n}(T)=\left(x \mid x, T x, \cdots, I^{n-1} x \in U(T)\right)
$$

by the formula

$$
f(i) x=\frac{(a l-T)^{n}}{2 \pi i} \int_{\Gamma} \frac{f(\lambda) R_{i}(T) x}{(\alpha-\lambda)^{n}} d \lambda
$$

where $\alpha$ is any point exterior to $\Gamma$. Equivalently,

$$
f(1) x=\sum_{i=0}^{n-1} \frac{f^{(l)}(0)}{i !} T^{i} x+\frac{1}{2 \pi i} \int_{\Gamma} \frac{f(\lambda) R_{\lambda}(T) T_{x}^{n}}{\lambda^{n}} d \lambda
$$

The usual homomorphism rules hold, and the results are consistent with those of Taylor. A closed extension of $f(T)$ is obtained which coincides with the Stone-von Neumann operator in the case of self-adjoint $T$ in Hilbert space.

In $\delta 5$ we assume a further growth condition on $\left\|R_{\lambda}(T)\right\|$ near $\sigma(T)$, and investigate operators corresponding to bilateral transforms. This section is largely a reformulation for our situation of results of Hille [3, Chap. 15] for an operational calculus for the case that $\sigma(T)$ is confined to a half plane and $f$ is 
a one-sided transform. In $\S 7$ we prove a theorem on the construction of inverses of such operators by limits of polynomials in $T$.

As an application we take $T$ the operation of differentiation in the spaces $C$ and $L_{p}(1 \leq p<\propto)$ on the real line and the unit circle. For the case of the line where $\sigma(T)$ is the imaginary axis, if

$$
f(\lambda)=\int_{-\infty}^{\infty} e^{\lambda \xi} G(\xi) d \xi
$$

converges absolutely in a strip containing $\sigma(T)$, then

$$
f(T) x(t)=\int_{-\infty}^{\infty} G(\xi) x(t-\xi) d \xi .
$$

Consider the Stielties transform

$$
\dot{\phi}(t)=\frac{1}{\pi} \int_{-\infty}^{\infty} \operatorname{sech} \frac{\xi}{2} x(t-\xi) d \xi
$$

for which $f(\lambda)=[\cos \pi \lambda]^{-1}$. Writing

$$
\cos \pi \lambda=\prod_{k=1}^{\infty}\left(1-\frac{\lambda^{2}}{(k-1 / 2)^{2}}\right)
$$

we see from the inversion theorem of $\S 7$ that

$$
\lim _{n \rightarrow \infty} \prod_{k=1}^{n}\left(1-\frac{1}{(k-1 / 2)^{2}} \frac{d^{2}}{d t^{2}}\right) \phi(t)=x(t)
$$

where convergence is in the norm topology of any of the spaces mentioned. This example is typical of a class of inversion theorems for which the theorem of $\$ 7$ gives a uniform treatment. Inversion formulas of this sort have been proved by different methods for $L_{2}(-\infty, \infty)$ by Pollard [9] and for $C[-\infty, \infty)$ by Widder [16] (see Hirschman and Widder [4, 5,6 and 7] for extensive ratults on the corresponding local problem). The case $p \neq 2$ does not seem to have been considered before. Our method also applies to inversion of transforms

$$
\int_{-\pi}^{\pi} H(\xi) x(t-\xi) d \xi
$$

in the corresponding spaces on the circle. 
2. Construction of the calculus. Let $T$ be a closed operator whose domain $\mathcal{L}(T)$ is a prescribed subspace. Te suppose:

A. The spectrum $\sigma\left(T^{\prime}\right)$ lies in the vertical strip

$$
-\gamma \leq \sigma \leq \gamma \quad(\lambda=\sigma+i \tau, 0 \leq \gamma<\propto) .
$$

3. The resolvent $k_{\lambda}(T)=(\lambda I-T)^{-1}$ satisfies

$$
\left\|R_{\lambda}(T)\right\| \leq M(t),|\sigma| \geq t, t>\gamma^{1}
$$

The strip containing $\sigma(T)$ is taken to be vertical merely for convenience. The symbol $\rho(T)$ will denote the resolvent set, and $[X]$ the set of bounded linear operators mapping $X$ into itself. We shall need the following known results:

(a) As $T$ is closed, $k_{\lambda}(T)$ is in $[X]$ for $\lambda \in \rho(T)[14, p .110]$.

(b) If $L_{n}(T)$ are the subspaces defined by

$$
H_{g}(T)=X, D_{n}(T)=\left(x \mid x, T x, \ldots, T^{n-1} x \in L(T)\right) \quad(n \geq 1),
$$

then for any polynomial

$$
P(\lambda)=a_{0} \lambda^{n}+a_{1} \lambda^{n-1}+\cdots+a_{n} \quad\left(a_{0} \neq 0\right),
$$

the corresponding operator $P(T)$ with domain $I_{n}(T)$ is closed, and $[14, \mathrm{p} .202]$.

$$
R_{\lambda}(T) P(T) x=P(T) R_{\lambda}(T) x
$$

(c) If $x \in D_{n}(T)$, and $\alpha$ is any complex number, then

$$
R_{\lambda}(T)=\sum_{i=0}^{n} \frac{(T-\alpha I)^{i} x}{\left(\lambda-\alpha_{1}\right)^{i+1}}+\frac{(T-\alpha I)^{n+1} R_{\lambda}(T) x}{(\lambda-\alpha)^{n+1}},
$$

and for any $m$ and $n, R_{\lambda}^{m}(T)$ maps $D_{n}(T)$ one-to-one onto $D_{m+n}(T)[14$, p. 204$205]$.

We shall also need the following elementary consequence of the definition

${ }^{1}$ One may show through the Phragmen-Lindelöf theorem (the proof in $[15, \mathrm{p} .177]$ holds for operator-valued functions) that $\mathrm{B}$ is implied by the apparently much weaker condition $\left\|R_{\lambda}(T)\right\|=O\left(e^{\beta|\sigma|}\right), \beta<1$. 
of a closed operator.

Lemma 2.1. Let $K$ be closed with domain $D$, and let

$$
H_{n} \in[X] \quad(n=1,2, \ldots),
$$

uith

$$
H x=\lim _{n \rightarrow \infty} I !_{n} x
$$

defined for each $x \in \lambda$. If $x \in D$, and

$$
H H_{n} K x=K H_{n} x
$$

for each $n$, then $H x \subset D$ and $K H x=H K x$.

Our procedure for assigning operators $f(T)$ to functions $f(\lambda)$ will be a variant of the contour integral approach of Dunfor 1 and Taylor. It will be convenient to set up the correspondence first for a particular class of functions and use this class to treat less restrictive cases.

DEFINITION 2.1. We denote by $\mathcal{f}(0, \gamma)$ the class of functions $f$ satisfyin:

(a) $f$ is analytic in a strip $-r<\sigma<r, r>\gamma$ ( $r$ may vary with $f$ ).

(b) As $\tau \longrightarrow \pm \infty, f(\sigma+i \tau) \longrightarrow 0$ uniformly with respect to $\sigma, \cdots r_{1} \leq \sigma \leq r_{1}$ for any $r_{1}<r$.

$$
\int_{-\infty}^{\infty}|f(\sigma+i \tau)| d \tau<\infty \quad(-r<\sigma<r) .
$$

The class $\mathcal{L}(0, \gamma)$ is an algebra, although strictly speaking not an algebra of functions, since the functions $f$ do not have a common domain. To get condition (c) for products, we note that if $f \in \mathcal{L}(0, \gamma), f(\sigma+i \tau)$ is bounded in $\tau$ for fixed $\sigma(-r<\sigma<r)$. Thus if $\left|f_{1}(\sigma+i \tau)\right| \leq M$ ( $\sigma$ fixed $)$,

$$
\int_{-\infty}^{\infty}\left|f_{1}(\sigma+i \tau) f_{2}(\sigma+i \tau)\right| \leq M \int_{-\infty}^{\infty}\left|f_{2}(\sigma+i \tau)\right| d \tau<\infty
$$

For convenience we adopt a convention with regard to contour integrals. If $f$ is analytic in $-r<\sigma<r$, where $r>\gamma$, the symbol $\Gamma_{c}(\omega)$ will denote a contour composed of the two parallel line segments $\sigma= \pm c,-\omega \leq \tau \leq \omega$, where $\gamma<c<r$; the positive sense along $\sigma=c$ will be upward, and that along $\sigma=-c$ 
downward. The symbol $\Gamma_{c}$ will denote the contour obtained by letting $\omega \longrightarrow \infty$.

We now define operators corresponding to functions in $\mathcal{L}(0, \gamma)$.

DEFINTION 2.2. For $f \in \mathcal{L}(0, \gamma)$, we set

$$
f(T) x=\frac{1}{2 \pi i} \int_{\Gamma_{c}} f(\lambda) R_{\lambda}(T) x d \lambda .
$$

This formula defines an operator in $[X]$, the integral converging absolutely and uniformly in $x$. It is easily seen to be independent of $c$ except for the restriction $y<c<r$.

THEOREM 2.1. If $f_{z} g \in \mathcal{L}(0, \gamma)$, then

(a) $(f+g)(T)=f(T)+g(T)$,

(b) $(f g)(T)=f(T) g(T)$.

Proof. Statement (a) is obvious. To prove (b) let $f$ and $g$ both satisfy the the conditions of Definition 2.1 in the strip $[-r . r]$, and let $c$ and $c^{\prime}$ be chosen so that $\gamma<c<c^{\prime}<r$. Using the functional equation

$$
R_{\lambda}(T)-R_{\mu}(T)=(\mu-\lambda) R_{\lambda}(T) R_{\mu}(T),
$$

we see readily that $f(T) g(T)$ is given by the expression

$$
\begin{aligned}
\frac{1}{2 \pi i} \int_{\Gamma_{c}} f(\lambda) R_{\lambda}(T) d \lambda & \frac{1}{2 \pi i} \int_{\Gamma_{c^{\prime}}} \frac{g(\mu)}{\mu-\lambda} d \mu \\
& +\frac{1}{2 \pi i} \int_{\Gamma_{c^{\prime}}} g(\mu) R_{\mu}(T) d \mu \frac{1}{2 \pi i} \int_{\Gamma_{c}} \frac{f(\lambda)}{\lambda-\mu} d \lambda .
\end{aligned}
$$

Since $c<c^{\prime}$,

$$
\frac{1}{2 \pi i} \int_{\Gamma_{c}} \frac{f(\lambda)}{\mu-\lambda} d \lambda=0, \frac{1}{2 \pi i} \int_{\Gamma_{c},} \frac{g(\mu)}{\mu-\lambda} d \mu=g(\lambda) .
$$

Formula (b) now follows.

Theorem 2.2. Let $f \in \mathcal{L}(0, \gamma), x \in D_{n}(T)$, and $P(T)$ be a polynomial in $T$ of degree $n$. Then $f(T) x \in D_{n}(T)$, and 


$$
P(T) f(T) x=f(T) P(T) x .
$$

Proof. For fixed $m$ let $\pi(i, m) \quad(i=1,2, \ldots)$ be a sequence of partitions of the contour $\Gamma_{c}(m)$ whose meshes go to zero as $i \longrightarrow \propto$. Setting

$$
H_{i m} x=\sum_{\pi(i, m)} f\left(\lambda_{k}\right)\left(\lambda_{k}-\lambda_{k-1}\right) R_{\lambda_{k}}(T) x,
$$

we have

$$
P(T) H_{i m} x=H_{i m} P(T) x
$$

for each $i$. Now letting $i \longrightarrow \infty$, we see from Lemma 2.1 that

$$
H_{m} x=\frac{1}{2 \pi i} \int_{\Gamma_{c}(m)} f(\lambda) R_{\lambda}(T) x d^{d} \lambda \in D_{n}(T),
$$

and

$$
P(T) H_{m} x=H_{m} P(T) x .
$$

We now apply the lemma again as $m \longrightarrow \propto$.

Tie note also the following useful consequence of (2.3).

LEMMA 2.2. Let $f \in \mathcal{L}(0, \gamma)$ and $|\mathcal{R}(\alpha)|>c$. Then

$$
k_{\alpha}(T) f(T) x=\frac{1}{2 \pi i} \int_{\Gamma_{c}} \frac{f(\lambda) l_{\lambda}(T) x}{\alpha-\lambda} d \lambda .
$$

To assign operators to functions with less restrictive growth properties than those of $\mathcal{L}(0, \gamma)$, we must overcome the problem of the convergence of the integral in (2.2). As motivation, suppose that $f \in \mathcal{L}(0, \gamma)$ and $x \in \ddot{D}_{n}(T)$. Then, by the Lemma 2.2 ,

$$
R_{\alpha}^{n}(T) f(T) x=\frac{1}{2 \pi i} \int_{\Gamma_{c}} \frac{f(\lambda) k_{\lambda}(T) x}{(\alpha-\lambda)^{n}} d \lambda,
$$

or, by use of Theorem 2.2,

$$
f(T) x=\frac{1}{2 \pi i} \int_{\Gamma_{c}} \frac{f(\lambda) k_{\lambda}^{2}(T)\left(c_{1} I-T\right)^{n} x}{(\alpha-\lambda)^{n}} d \lambda .
$$

The convergence-producing factor in the denominator suggests the following development. 
DEFINITION 2.3. For each $n \geq 0$, let

$$
\mathcal{L}(n, \gamma)=\left(f \mid f(\lambda)(\alpha-\lambda)^{-n} \in \mathcal{L}(0, \gamma) \text { for }|R(a)|>\gamma\right)
$$

and let $\mathcal{L}(\propto, \gamma)=\cup_{n=0}^{\infty} \mathcal{L}(n, \gamma)$.

The definition of $\mathcal{L}(n, \gamma)$ does not depend on $\alpha$, since if $f \in \mathcal{L},(n, \gamma)$ for one $\alpha$ it is in for any other with $|R(\alpha)|>\gamma$. We note that

$$
\mathcal{L}(0, \gamma) \subset \mathcal{L}(1, \gamma) \subset \cdots
$$

$\mathcal{L}(\infty, \gamma)$ is an algebra.

THEOREM 2.3. If $f, g \in \mathcal{L}(n, \gamma)$, then $\alpha f+\beta g \in \mathcal{L}(n, \gamma)$. If $f \in \mathcal{L}(m, \gamma)$ and $g \in \mathcal{L}(n, \gamma)$, then $f g \in \mathcal{L}(m+n, \gamma)$.

We omit the proof, which follows from the fact $\mathcal{L}(0, \gamma)$ is an algebra.

DEFINITION 2.4. For $f \in \mathcal{L}(n, \gamma)$, and $x$ in $D_{n}(T)$, we choose $\alpha$ such that $|R(\alpha)|>\gamma$, and define

$$
f(T) x=\frac{1}{2 \pi i} \int_{\Gamma_{c}} \frac{f(\lambda) R_{\lambda}(T)(\alpha . I-T)^{n} x}{(\alpha-\lambda)^{n}} d \lambda
$$

where $c<|R(a)|$.

To show that this definition is independent of $\alpha$, let $f \in \mathcal{L}(n, \gamma), n \geq 1$, $x \in L_{n}(T)$, and $|R(\alpha)|,|R(\beta)|>c$. By Lemma 2.2,

$$
\begin{aligned}
& R_{\alpha}^{n}(T) \frac{1}{2 \pi i} \int_{\Gamma_{c}} \frac{f(\lambda) R_{\lambda}(T)(\beta I-T)^{n} x}{(\beta-\lambda)^{n}} d \lambda \\
& \quad=\frac{1}{2 \pi i} \int_{\Gamma_{c}} \frac{f(\lambda) R_{\lambda}(T)(\beta I-T)^{n} x}{(\beta-\lambda)^{n}(\alpha-\lambda)^{n}} d \lambda \\
& =\frac{1}{2 \pi i} \int_{\Gamma_{c}} \frac{f(\lambda) R_{\lambda}(T) R_{\beta}^{n}(T)(\beta I-T)^{n} x}{(\alpha-\lambda)^{n}} d \lambda \\
& =K_{\alpha}^{n}(T) \frac{1}{2 \pi i} \int_{I_{c}} \frac{f(\lambda) R_{\lambda}(T)(\alpha I-T)^{n} x}{(\alpha-\lambda)^{n}} d \lambda .
\end{aligned}
$$


Since $R_{\alpha}^{n}(T)$ has an inverse,

$$
\frac{1}{2 \pi i} \int_{\Gamma_{c}} \frac{f(\lambda) i_{\lambda}(T)(\alpha I-T)^{n} x}{(\alpha-\lambda)^{n}} d \lambda=\frac{1}{2 \pi i} \int_{\Gamma_{c}} \frac{f(\lambda) R_{\lambda}(T)(\beta I-T)^{n} x}{(\beta-\lambda)^{n}} d \lambda
$$

We remark also that when $x \in \ldots(n, y)$, it is also in $\alpha(n+1, y)$; and if $x \in D_{n+1}(7)$, Iemma 2.2 shows that formula 2.1 for both $n$ and $n+1$ yields the same operator.

THEOREM 2.4. Let $f \in \mathcal{L}(m, \gamma), g \in \mathcal{L}(n, \gamma)$, and $x \in L_{m+n}(T)$. Then $g(T) x \in D_{n}(T)$, and $f(T) g(T) x=(f g)(T) x$.

Proof. We note that

$$
\left.f_{g} \in \mathcal{L}(m+n, \gamma) \text { and } \xi(l) \in \in L_{n !} ! ?\right)
$$

by Theorems 2.2 and 2.3. Now if we write

$$
h(\lambda)=f(\lambda)(\alpha-\lambda)^{-m}, k(\lambda)=g(\lambda)(\alpha-\lambda)^{-n} \text {, and }(\alpha)>\lambda
$$

then, by The orems 2.1 and 2.2,

$$
\begin{aligned}
f(T) g(T) x & =h(T)(\alpha I-T)^{m} k(T)\left(\dot{L} I-\frac{n}{i}\right)^{n} x \\
& =(\alpha I-T)^{m+n} h(T) k(T) x \\
& =(\alpha I-T)^{m+n}(h k)(T) x=(f g)(T) x .
\end{aligned}
$$

We are led to another formula for $f(T)$ in the following way. Suppose first that $f \in \mathcal{L}(0, \gamma)$ and $x \in D_{n}(T)$. Then in the integral (2.2) we may substitute

$$
R_{\lambda}(T) x=\sum_{i=0}^{n-1} \frac{T^{i} x}{\lambda^{i+1}}+\frac{k_{\lambda}(T) T^{n} x}{\lambda^{n}}
$$

to obtain ${ }^{2}$

${ }^{2} \mathrm{~A}$ formula of this type for $n=2$ is used by Hille [3, p. 239]. 


$$
f(T) x=\sum_{i=0}^{n-1} \frac{f^{(i)}(0) T^{i} x}{i !}+\frac{1}{2 \pi i} \int_{\Gamma_{c}} \frac{f(\lambda) R_{\lambda}(T) T^{n} x}{\lambda^{n}} d \lambda .
$$

This formula has meaning when $f \in \mathcal{L}(n, \gamma)$ instead of $\mathcal{L}(0, \gamma)$, and we shall establish it. In fact we prove more generally:

THEOREM 2.5. Let $f \in \mathcal{L}(n, \gamma)$ and $x \in D_{n}(T)$. If $|R(\alpha)|<c$, then

$$
f(T) x=\sum_{i=0}^{n-1} \frac{f^{(i)}(\alpha)(T-\alpha I)^{i} x}{i !}+\frac{1}{2 \pi i} \int_{\Gamma_{c}} \frac{f(\lambda) R_{\lambda}(T)(T-\alpha I)^{n} x}{(\lambda-\alpha)^{n}} d \lambda
$$

Proof. We suppose first that $|R(\alpha)|>\gamma$, and choose $c^{\prime}$ such that $\gamma<c^{\prime}<$ $|R(\alpha)|<c$. Then

$$
\begin{aligned}
f(T)=\frac{1}{2 \pi i} \int_{\Gamma_{c},} \frac{f(\lambda) R_{\lambda}(T)(T-\alpha I)^{n} x}{(\lambda-\alpha)^{n}} d \lambda \\
\\
\quad-\frac{1}{2 \pi i} \int_{C} \frac{f(\lambda) R_{\lambda}(T)(T-\alpha I)^{n} x}{(\lambda-\alpha)^{n}} d \lambda,
\end{aligned}
$$

where $C$ is a small circle described counterclockwise enclosing $\alpha$ and not intersecting $\Gamma_{c}$ or $\Gamma_{c}{ }^{\prime}$. Substituting from $(2.1)$ in the second integral, we have

$$
\begin{aligned}
-\frac{1}{2 \pi i} & \int_{C} \frac{f(\lambda) R_{\lambda}(T)(T-\alpha I)^{n} x d \lambda}{(\lambda-\alpha)^{n}} \\
& =\frac{1}{2 \pi i} \int_{C} f(\lambda) R_{\lambda}(T) x d \lambda+\sum_{k=0}^{n-1} \frac{1}{2 \pi i} \int_{C} \frac{f(\lambda)}{(\lambda-\alpha)^{k}} d \lambda(T-\alpha I)^{k} x \\
& =\sum_{k=0}^{n-1} \frac{f^{(k)}(\alpha)}{k !}(T-\alpha I)^{k} x .
\end{aligned}
$$

This establishes (2.5) when $|R(\alpha)|>\gamma$. However, the right side of (2.5) is independent of $\alpha$ and analytic in $\alpha$. Thus (2.5) holds in the larger region.

The calculus developed above has the disadvantage that if $P(\lambda)$ is a polynomial of degree $n$, and hence is at best in $\mathcal{L}(n+2, \gamma)$, the operator corre- 
sponding to $P(\lambda)$ is defined only on $D_{n+2}(T)$. We check that this operator is really the formal polynomial in $T$ in the typical case $f(\lambda)=\lambda$. For $x$ in $D_{3}(T)$, using the series formula, we have

$$
f(T) x=T x+\frac{1}{2 \pi i} \int_{\Gamma_{c}} \frac{R_{\lambda}(T) T^{3} x}{\lambda^{2}} d \lambda,
$$

since $f(0)=f^{\prime \prime}(0)=0$. But

$$
\begin{aligned}
\left\|\int_{\Gamma_{c}} \frac{R_{\lambda}(T) T^{3} x}{\lambda^{2}} d \lambda\right\| & \leq 2 M(c)\left\|T^{3} x\right\| \int_{-\infty}^{\infty} \frac{d \tau}{c^{2}+\tau^{2}} \\
& =\frac{2 \pi\left\|T^{3} x\right\| M(c)}{c} .
\end{aligned}
$$

The left side is independent of $c$, and must vanish since $M(c)$ is nonincreasing.

In passing we note without proof some facts which we shall not need later. If for $x \in D_{n}(T)$ we write

$$
\|x\|_{n}=\sum_{i=0}^{n}\left\|T^{i} x\right\|
$$

then $D_{n}(T)$ with this norm is a Banach space $X^{(n)}$; and if $f \in \mathcal{L}(n, \gamma)$, the operator $f(T)$ defined on $X^{(n)}$ to $X$ is bounded. If $D(T)$ is dense in $X$ (a condition of $\S 5)$, then $D_{m}(T)$ is dense in $X^{(n)}$ for $m>n$.

3. Consistency with Taylor's operators. For an arbitrary closed operator with nonempty resolvent set, Taylor [14] has defined an operator corresponding to any function $f$ analytic on $\sigma(T)$ and at infinity. In our situation, let us denote by $G(\gamma)$ the class of functions whose singularities lie in bounded sets in the two half-planes $\sigma<-\gamma$ and $\sigma>\gamma$. If $f \in G(\gamma)$, and $\Omega_{1}$ and $\Omega_{2}$ are clockwise contours in $\rho(T)$ enclosing these sets, then Taylor's formula defines

$$
f[T]=f(\propto) I+\frac{1}{2 \pi i} \int_{\Omega_{1}+\Omega_{2}} f(\lambda) R_{\lambda}(T) d \lambda .
$$

This operator is bounded, and the correspondence $f \longrightarrow f[T]$ preserves sums and products. In our theory, $\mathcal{G}(\gamma) \subset \mathcal{L}(2, \gamma)$; and the corresponding operator 
$f(T)$ are defined only on $D_{2}(T)$. We shall show that $f[T]$ is an extension of $f(T)$. First we note the relationship between $\mathcal{G}(\gamma)$ and the classes $\mathcal{L}(n, \gamma)$.

Lemma 3.1. Let $f \in \mathcal{G}(\gamma)$. Then $f \in \mathcal{L}(k, \gamma)(k=0,1)$ if and only if it has a zero of order at least $2-k$ at infinity.

Proof. First let $f \in \mathcal{L}(0, \gamma) \cap \mathcal{G}(\gamma)$, and let $R$ be the radius of a circle about the origin containing the singularities of $f$ in its interior. Since clearly $f(\infty)=0$, we may write

$$
f(\lambda)=\frac{a}{\lambda}+\frac{g(\lambda)}{\lambda^{2}}
$$

where $g$ is analytic, and

$$
|g(\lambda)|<M \text { for }|\lambda| \geq R
$$

Then if $a \neq 0$,

$$
\int_{R}^{n}|f(i \tau)| d \tau \geq|a| \log \frac{n}{R}-M \int_{R}^{n} \frac{d \tau}{\tau^{2}},
$$

or

$$
\lim _{n \rightarrow \infty} \int_{R}^{n}|f(i \tau)| d \tau=\infty
$$

which contradicts Condition (C) of Definition 2.1. Thus $f$ has a zero of order two at infinity. The converse is clear. For the case $k=1$, we use the foregoing argument on $f(\lambda)(\alpha-\lambda)^{-1}$.

THеовем 3.1. Let $f \in \mathcal{L}(0, \gamma) \cap \mathcal{G}(\gamma)$. Then $f(T)=f[T]$.

Proof. Let $K$ be a large circle of radius $r$ centered at zero, and let $c$ be chosen $(c>\gamma)$ so that the singularities of $f$ lie interior to $K$ but exterior to $\Gamma_{c}$. Let

$$
\Gamma_{c}(\omega)
$$$$
\left(\omega=\sqrt{r^{2} c^{2}}\right)
$$

denote that part of $\Gamma_{c}$ cut off by $K$, and let $S$ be the two arcs of $K$ (described counterclockwise) which lie exterior to $\Gamma_{c}$. In formula (4.1), for $f[T]$ we may take 


$$
\Omega_{1}+\Omega_{2}=S+\Gamma_{c}(\omega)
$$

Now, by Lemma 3.1,

$$
|f(\lambda)|=O\left(\lambda^{-2}\right)
$$

for large values of $\lambda$. Also by Condition $\mathrm{B}$ of $\xi 2,\left\|R_{\lambda}(T)\right\|$ is bounded if $|\sigma| \geq c$. Thus

$$
\left\|\int_{S} f(\lambda) R_{\lambda}(T) d \lambda\right\|=O\left(\frac{1}{r}\right)
$$

As

$$
\lim _{r \rightarrow \infty} \Gamma_{c}(\omega)=\Gamma_{c}
$$

the theorem is proved.

Corollary 3.1. If $f \in G(\gamma)$, then

$$
f[T] x=f(T) x
$$

for $x$ in $D_{2}(T)$.

Proof. Let

$$
g(\lambda)=f(\lambda)(\alpha-\lambda)^{-2}
$$

Then

$$
g \in \mathcal{L}(0, \gamma) \cap \mathcal{G}(\gamma) \text { and } g[T]=g(T) \text {. }
$$

But (see [14, p. 203])

$$
f(T) x=(\alpha I-T)^{2} g(T)=\left(c_{I} I-T\right)^{2} g[T]=f[T] .
$$

Similarly one shows if $f \in \mathcal{L}(1, \gamma) \cap \mathcal{G}(\gamma)$ then

$$
f(T) x=f[T] x
$$

for $x$ in $D(T)$.

4. A closed extension of $f(T)$. Let $f$ be in $\mathcal{L}(n, \gamma)$, but not in $\mathcal{L}(n-1, \gamma)$. 
The operator $f(T)$, defined on $D_{n}(T)$, need not in general be closed on this domain. However, we can describe an extended domain on which it will be closed. As before, we set

$$
h(\lambda)=f(\lambda)(\alpha-\lambda)^{-n},|R(\alpha)|>\gamma,
$$

and write

$$
f(T) x=(\alpha I-T)^{n} h(T) x .
$$

DEFINITION 4.1. We define

$$
D(\tilde{f}(T))=\left(x \mid h(T) x \in D_{n}(T)\right),
$$

and define

$$
\tilde{f}(T) x=(\alpha I-T)^{n} h(T) x
$$

for $x$ in $D(\tilde{f}(T))$.

Since $h \in \mathcal{L}(0, \gamma), D_{n}(T) \subset D(\tilde{f}(T))$ by Theorem 2.2. The inclusion may be proper. We shall need a lemma.

LEMMA 4.1. Let $f$ in $G(\gamma)$ have a zero of order $n($ but not $n+1)$ at infinity. Then $x \in D_{m}(T)(m \geq 0)$ if and only if $f[T] x \in D_{m+n}(T)$.

Proof. Necessity is proved by Taylor [14, p. 203]. To prove sufficiency first suppose that $n=0$, i.e., $f(\infty) \neq 0$, and that

$$
f[T] x \in D_{m}(T) .
$$

If $x \in D_{k}(T)$, where $0 \leq k<m$, then

$$
\begin{aligned}
f[T] x & =f(\infty) x+\frac{1}{2 \pi i} \int_{\Omega_{1}+\Omega_{2}} f(\lambda) R_{\lambda}(T) x d \lambda \\
& =f(\infty) x+h[T] x,
\end{aligned}
$$

where $h(\lambda)=f(\lambda)-f(\infty)$. As $h$ has a zero at infinity, $h[T] x \in D_{k+1}(T)$ by the first half of the lemma. But as $k<m$,

$$
f[T] x \in D_{k+1}(T) \subset D_{m}(T),
$$


and hence $x \in D_{k+1}(T)$. By repeating the argument, we see that $x \in D_{m}(T)$. If $n \geq 1$ and

$$
f[T] x \in D_{m+n}(T),
$$

we set

$$
g(\lambda)=(\alpha-\lambda)^{n} f(\lambda)
$$

and note that

$$
f[T] x=R_{\alpha}^{n}(T) g[T] x .
$$

As $R_{\alpha}^{n}(T)$ maps $D_{m+n}(T)$ one-to-one onto $D_{m}(T)$,

$$
g[T] x \in D_{m}(T) .
$$

But since $g(\infty) \neq 0, x \in L_{m}(T)$ by the case $n=0$ just proved.

THEOREM 4.1. The subspace $D(\widetilde{f}(T))$ is independent of $\alpha_{\text {, and }} \widetilde{f}(T)$ with domain $D(\widetilde{f}(T))$ is a closed extension of $f(T)$.

Proof. If $|R(\alpha)|,|R(\beta)|>\gamma, \alpha \neq \beta$ we denote by $D_{\alpha}(\tilde{f}(T)), D_{\beta}(\tilde{f}(T))$, $h_{\alpha}$, and $h_{\beta}$ the respective domains and functions $f(\lambda)(\alpha-\lambda)^{-n}$ and $f(\lambda)(\beta-\lambda)^{-n}$. Then

$$
h_{\alpha}(\lambda)-h_{\beta}(\lambda)=\frac{P(\lambda) h_{\beta}(\lambda)}{(\alpha-\lambda)^{n}}=g(\lambda) h_{\beta}(\lambda),
$$

where $P$ is a polynomial of degree $n-1$. The function $g$ is in $G(\gamma)$, and $g[T]$ carries $D_{n}(T)$ into itself (in fact into $D_{n+1}(T)$ ) by the lemma. Since

$$
\left(g h_{\beta}\right)(T)=g[T] h_{\beta}(T)
$$

(apply $R_{\beta}(T)$ to both sides),

$$
h_{\alpha}(T)=h_{\beta}(T)+g[T] h_{\beta}(T)
$$

and

$$
D_{\beta}(\widetilde{f}(T)) \subset D_{\alpha}(\tilde{f}(T)) .
$$


Symmetry gives the reverse. Now fixing $a$ and associated $h(\lambda)$, let

$$
x_{n} \in L(\tilde{f}(T)) \quad(n=1,2, \cdots),
$$

and suppose

$$
\lim x_{m}=x_{0} \text { and } \lim \tilde{f}(T) x_{m}=y_{0} \cdot
$$

Then

$$
\lim h(T) x_{m}=h(T) x_{0},
$$

as $h(T)$ is continuous. As $h(T) x_{m} \in D_{n}(T)$, and $(\alpha I-T)^{n}$ is closed on $D_{n}(T), h(T) x_{0} \in D_{n}(T)$; i.e. $x_{0} \in D(\widetilde{f}(T))$, and

$$
\widetilde{f}(T) x_{0}=(\alpha l-T)^{n} h(T) x_{0}=y_{0} .
$$

When

$$
f(\lambda)=\sum_{i=0}^{n} a_{i} \lambda^{i}
$$

the operator $\tilde{f}(T)$ is the formal polynomial in $T$. For if

$$
h(\lambda)=f(\lambda)(\alpha-\lambda)^{-(n+2)}
$$

then $h \in G(\gamma)$, with a zero of order exactly two at infinity. By Lemma 4.1,

$$
h[T] x(=h(T) x) \in J_{n+2}(T)
$$

if and only if $x \in D_{n}(T)$. Hence

$$
D_{n}(T)=L(\tilde{f}(T))
$$

If $x \in D_{n}(T)$,

$$
R_{\alpha}^{n+2}(T) \tilde{f}(T) x=h[T] x=R_{\alpha}^{n+2}(T) \sum_{i=0}^{n} a_{i} T^{i} x,
$$

so

$$
\tilde{f}(T)=\sum_{i=0}^{n} a_{i} T^{i}
$$


By similar reasoning one shows that $\tilde{f}(T)=f[T]$ for $f \in \mathcal{G}(\gamma)$.

We now identify $\tilde{f}(T)$ in the case that $X$ is a Hilbert space and $A=-i T$ is self-adjoint $[8, \mathrm{p} .44]$. If $\left\{E_{\tau}\right\}(-\infty<\tau<\infty)$ is the resolution of $I$ associated with $A, D(A)=D(T)$ is the set of $x$ for which

$$
\lim _{k \rightarrow \infty} \int_{-\infty}^{\infty} \tau e_{k}(\tau) d E_{\tau} x
$$

exists, where $e_{k}(\tau)$ is one if $-k \leq \tau \leq k$ and zero otherwise. If $F(\tau)$ is continuous, we define $D_{F}$ as the set of $x$ for which

$$
F(A) x=\lim _{k \rightarrow \infty} \int_{-\infty}^{\infty} F(\tau) e_{k}(\tau) d E_{\tau} x
$$

exists. The operator $F(A)$ is normal, and hence closed on $D_{F}$. We write

$$
D_{F}=D\left(A^{n}\right) \text { if } F(\tau)=\tau^{n}
$$

Taking $F(\tau)=f(i \tau)$, we easily see that $f(T)=F(A)$ when $f \in \mathcal{L}(0, \gamma)$ (here $\gamma=0$ ) and that $D\left(A^{n}\right)=D_{n}(T)$. Now let $f \in \mathcal{L}(n, \gamma)$,

$$
h(\lambda)=f(\lambda)(\alpha-\lambda)^{-n},|R(\alpha)|>0, \text { and } H(\tau)=h(i \tau)
$$

If

$$
h(T) x \in D_{n}(T),
$$

that is

$$
H(A) x \in D\left(A^{n}\right)=D\left((\alpha I-i A)^{n}\right),
$$

then

$$
\begin{aligned}
\lim _{k \rightarrow \infty} & \int_{-\infty}^{\infty} F(\tau) e_{k}(\tau) d E_{\tau} x \\
& =\lim _{k \rightarrow \infty} \int_{-\infty}^{\infty}(\alpha-i \tau)^{n} e_{k}(\tau) d E_{\tau} \int_{-\infty}^{\infty} H(\mu) d E_{\mu} x
\end{aligned}
$$

exists and $x \in D_{F}$. The argument may be reversed. Thus 


$$
D_{F}=\bar{D}(\widetilde{f}(T))
$$

and

$$
F(A)=(\alpha I-i A) H(A)=(\alpha I-T)^{n} h(T)=\tilde{f}(T) .
$$

5. Operators corresponding to bilateral transforms. A class of operators of particular interest in applications is the set corresponding to the subclass $U(\gamma) \subset \mathcal{L}(2, \gamma)$ of absolutely convergent bilateral Laplace-Stieltjes transforms $\int_{-\infty}^{\infty} e^{-\lambda \xi} d \beta(\xi)$. Dy a well-known theorem of llamburger [17, p. 265], any function in $\mathcal{L}(0, \gamma)$ is in $U(\gamma)$. While functions in $\mathcal{L}(0, \gamma)$ yield bounded operators, functions from $U(\gamma)$ may yield unbounded operators in the absence of additional assumptions on $T$ and $R_{\lambda}(T)$. In this section and throughout the rest of the paper we shall assume

$$
B^{\prime}: \quad\left\|R_{\lambda}(T)\right\| \leq \frac{1}{|\sigma|-\gamma}
$$

$$
\mathrm{C}: D(T) \text { is dense in } X,{ }^{3}
$$

which will ensure boundedness. The results here are essentially due to Hille; in his book [3, Chap. 15] he constructs a calculus for operators with spectrum in the left half-plane, the class of functions being one-sided transforms converging absolutely in a half-plane containing the spectrum. The details of the construction in our case will differ sufficiently to justify giving an outline of the development. We take the following result from Hille.

Тнеовем 5.1. [3, p. 307, 322]. If $T$ is a closed operator satisfying A, B', and $\mathrm{C}$, the formulas

$$
J(\xi) x= \begin{cases}\frac{1}{2 \pi i}(C, 1)-\int_{r-i \infty}^{r+i \infty} e^{\lambda \xi} R_{\lambda}(T) x d \lambda & (\xi>0), \\ x & (\xi=0), \\ \frac{1}{2 \pi i}(C, 1)-\int_{-r+i \infty}^{-r-i \infty} e^{\lambda \xi} R_{\lambda}(T) x d \lambda & (\xi<0),\end{cases}
$$

\footnotetext{
${ }^{3}$ Condition $C$ implies $D_{n}(T)$ is dense for $n>1$. For if $\lambda \in \rho(T), D_{n}(T)=R_{\lambda}^{n-1}(T) D(T)$ is dense in $R_{\lambda}^{n-1}(T) X=D_{n}(T)$, and thus by a repetition of the argument is dense in $D(T)$, and hence in $X$. It will follow from Theorem $5.1[3, \mathrm{p} .228]$ that $D_{\infty}(T)=\bigcap_{n=1}^{\infty} D_{n}(T)$ is also dense.
} 
where $x \in X, r>\gamma$, define a group of bounded operators $\exists(\xi),-\infty<\xi<\infty$, where

$$
g(\xi+\eta)=g(\xi) \exists(\eta),\|\ni(\xi)\| \leq e^{|\xi| \gamma}
$$

$\exists(\xi)$ is strongly continuous in $\xi$, and, for each $x \in D(T)$,

$$
\lim _{\xi \rightarrow 0} \frac{\zeta(\xi) x-x}{\xi}=T x .
$$

$R_{\lambda}(T)$ has the representation

$$
R_{\lambda}(T) x=\left\{\begin{array}{lr}
\int_{0}^{\infty} e^{-\lambda \xi \Im}(\xi) x d \xi & (\sigma>\gamma), \\
-\int_{-\infty}^{0} e^{-\lambda \xi \Im}(\xi) x d \xi & (\sigma<-\gamma),
\end{array}\right.
$$

$x \in X$, the integrals converging absolutely.

The operator $\mathcal{J}(\xi)$ will prove to be $f(T)$ for $f(\lambda)=e^{\lambda \xi}$.

Let $\Psi$ denote the vector space of complex-valued functions $\beta$ satisfying:

(a) $\beta$ is normalized and of bounded variation on $(-\infty, \infty)$.

(b) $\beta(-\infty)=\lim _{\xi \rightarrow-\infty} \beta(\xi)=0$.

(c) There is an $r>\gamma(r$ depending on $\beta)$, such that $\int_{-\infty}^{\infty} e^{-\lambda \xi} d \beta(\xi)$ converges absolutely for $-r \leq \sigma \leq r$.

We denote by $\Psi_{c}$ the subclass of $\Psi$ of $\beta$ continuous at zero and write

$$
\begin{aligned}
& \Psi_{+}=\left(\beta \mid \beta \in \Psi_{c}, \beta(\xi)=0, \quad \xi \leq 0\right), \\
& \Psi_{-}=\left(\beta \mid \beta \in \Psi_{c}, \beta(\xi)=\beta(0), 0<\xi\right) .
\end{aligned}
$$

If $u$ denotes the function

$$
u(\xi)= \begin{cases}0 & (\xi<0), \\ \frac{1}{2} & (\xi=0), \\ 1 & (0<\xi),\end{cases}
$$


then each $\beta \in \Psi$ has a unique representation

$$
\beta=\beta_{+}+\beta_{-}+[\beta(0+)-\beta(0-)] u,
$$

where $\beta_{+} \in \Psi_{+}, \beta_{-} \in \Psi_{-}$. The latter functions are given by

$$
\beta_{+}(\xi)=\beta(\xi)-\beta(0+)
$$

and

$$
\beta_{-}(\xi)=\beta(\xi)
$$

and $\beta\left(0_{-}\right)$otherwise.

DeFinition 5.1. A function $f$ is in $V(\gamma)$ if and only if

$$
f(\lambda)=\int_{-\infty}^{\infty} e^{-\lambda \xi} d \beta(\xi),
$$

where $\beta \in \Psi$. It is in $G(\gamma)$ if and only if $f \in V(\gamma)$ and $\beta$ is absolutely continuous.

We write $f=f_{+}+f_{-}+\left[\beta(0+)-\beta\left(0_{-}\right)\right]$corresponding to the decomposition above, and note that

$$
f_{+}(\lambda)=\int_{-\infty}^{0} e^{-\lambda \xi} d \beta_{+}(\xi), f_{-}(\lambda)=\int_{0}^{\infty} e^{-\lambda \xi} d \beta_{-}(\xi)
$$

Thus $f_{+}$and $f_{-}$are analytic for $\sigma<r$ and $\sigma>-r$ respectively.

THEовем 5.2. If $f \in U(\gamma)$, then the bounded operator $f\{T\}$ defined by

$$
f\{T\} x=\int_{-\infty}^{\infty} \Im(-\xi) x d \beta(\xi)
$$

is also given by

$$
\begin{aligned}
f\{T\} x & =\frac{1}{2 \pi i}(C, 1)-\int_{r-i \infty}^{r+i \infty} f(\lambda) R_{\lambda}(T) x d \lambda \\
& +\frac{1}{2 \pi i}(C, 1)-\int_{-r+i \infty}^{-r-i \infty} f(\lambda) R_{\lambda}(T) x d \lambda .
\end{aligned}
$$


Moreover,

$$
\begin{aligned}
& f_{+}\{T\} x=\int_{-\infty}^{0} \rightrightarrows(-\xi) x d \beta_{+}(\xi)=\frac{1}{2 \pi i}(C, 1)-\int_{r-i \infty}^{r+i \infty} f_{+}(\lambda) R_{\lambda}(T) x d \lambda, \\
& f_{-}\{T\} x=\int_{0}^{\infty} \zeta(-\xi) x d \beta_{-}(\xi)=\frac{1}{2 \pi i}(C, 1)-\int_{-r+i \infty}^{-r-i \infty} f_{-}(\lambda) R_{\lambda}(T) x d \lambda
\end{aligned}
$$

and if $\beta=u, f\{T\} x=x$, the two integrals of (5.6) each yield $x / 2$. If $x \in D_{2}(T)$, $f\{T\} x=f(T) x$ in the sense of $\$ 2$.

We sketch the proof. Consider the first integral of (5.6). After substituting from (5.3) and (5.5), and interchanging the order of integration, we may express the partial integral

$$
\begin{gathered}
\frac{1}{2 \pi} \int_{-\omega}^{\omega}\left[1+\frac{|\tau|}{\omega}\right] f(r+i \tau) R_{r+i \tau}(T) x d \tau \\
=\int_{0}^{\infty} e^{-r \xi} v(\xi, \omega) d \beta_{+}(\xi)+\int_{-\infty}^{0} e^{-r \xi} v(\xi, \omega) d_{\beta_{-}}(\xi) \\
+[(\beta(0+)-\beta(0-)] v(0, \omega),
\end{gathered}
$$

where

$$
v(\xi, \omega)=\int_{-\infty}^{\infty} v(\alpha) \cdot \frac{2 \sin ^{2} \omega(\xi-\alpha) / 2 d \alpha}{\omega(\xi-\alpha)^{2}}
$$

and

$$
v(\alpha)= \begin{cases}e^{r a j}(-\alpha) x & (\alpha<0), \\ x / 2 & (\alpha=0), \\ 0 & (0<\alpha) .\end{cases}
$$

The classical theorem on the Fejer integral holds for vector-valued functions (see [3, p. 49]). Thus

$$
\lim _{n \rightarrow \infty} v(\xi, \omega)=v(\xi)
$$

for each $\xi$ and, in fact, uniformly in any bounded interval of continuity of $v(\xi)$. 
Since $\beta_{+}$and $\beta_{-}$are continuous at zero, one shows easily that the first integral on the right of (5.7) vanishes in the limit, and

$$
\begin{aligned}
\frac{1}{2 \pi i}(C, 1)-\int_{r-i \infty}^{r+i \infty} f(\lambda) R_{\lambda}(T) x d \lambda & =\int_{-\infty}^{0} \xi(-\xi) x d \xi+\frac{[\beta(0+)-\beta(0-)]}{2} x \\
& =f_{-}\{T\} x+\frac{[\beta(0+)-\beta(0-)]}{2} x .
\end{aligned}
$$

The second integral of (5.6) yields $f_{+}\{T\} x+\left[\beta\left(0_{+}\right)-\beta\left(0_{-}\right)\right] x$, and the sum of the two is $f\{T\} x$.

Finally, when $x \in L_{2}(T)$ we may substitute

$$
R_{\lambda}(T) x=\frac{x}{\lambda}+\frac{T x}{\lambda^{2}}+\frac{R_{\lambda}(T) T^{2} x}{\lambda^{2}}
$$

in (5.6). Calculation of residues yields

$$
f\{T\} x=f(0) x+f^{\prime}(0) T x+\frac{1}{2 \pi i} \int_{\Gamma_{r}} \frac{f(\lambda) R_{\lambda}(T) T^{2} x}{\lambda^{2}} d \lambda=f(T) x .
$$

It follows from the foregoing theorem that $\mathfrak{J}(\xi)$ is $f\{T\}$ for $f(\lambda)=e^{\lambda \xi}$. If $f_{1}, f_{2} \in U(\gamma)$, one shows easily [3, p. 309] that

$$
\left(f_{1} f_{2}\right)\{T\} x=\int_{-\infty}^{\infty} \exists(-\xi) x d \theta(\xi)=f_{1}\{T\} f_{2}\{T\} x,
$$

where

$$
\theta(\xi)=\int_{-\infty}^{\infty} \beta_{1}(\xi-\eta) d \beta_{2}(\eta)
$$

We also note that

$$
\|f\{T\}\| \leq \int_{-\infty}^{\infty} e^{|\xi| \gamma}|d \beta(\xi)|,
$$

and if $x \in D_{n}(T), f \in \cup(\gamma)$ then $f\{T\} x \in D_{n}(T)$. The proof of the latter follows that of Theorem 2.2.

6. A class of kernels. We shall denote by $G_{0}(\gamma)$ those functions in $G(\gamma)$ 
(see $\$ 3$ ) which vanish of infinity. Any $f$ in $G_{0}(\gamma)$ is in $G(\gamma)$, i.e., is an absolutely convergent bilateral Laplace transform. Our purpose is to characterize the kernels $G(\xi)$ for which

$$
f(\lambda)=\int_{-\infty}^{\infty} G(\xi) e^{-\lambda \xi} d \xi, f \in G_{0}(\gamma)
$$

For this we shall need certain well-known results.

An entire function $F$ is said to be of exponential type $\delta$ if

$$
\max _{|z|=r}|F(z)|=O\left(e^{(\delta+\epsilon) r}\right)
$$

for every positive $\epsilon$ and no negative $\epsilon$. Polya has shown [11, p. 585] that there is a one-to-one correspondence between entire functions of exponential type and functions analytic at infinity as follows: If

$$
f(\lambda)=\sum_{n=0}^{\infty} \frac{a_{n}}{\lambda^{n+1}}
$$

where $C$ is the natural radius of convergence, then

$$
f(\lambda)=\int_{0}^{\infty} e^{-\lambda \xi} F(\xi) d \xi
$$

where $F$ is the entire function

$$
F(\xi)=\sum_{n=0}^{\infty} \frac{a_{n} \xi^{n}}{n !}
$$

$F$ is of exponential type $C$. Conversely, $F$ determines $f$. Further let $K$ be the set of singularities of $f$, and

$$
k(\phi)=\max _{\lambda \in K} R\left(\lambda e^{-i \phi}\right)
$$

be its support function. The function

$$
h(\phi)=\varlimsup_{r \rightarrow \infty} \frac{\log \left|F\left(r e^{i \phi}\right)\right|}{r}
$$


is called the indicator of $F$ as it measures the growth of $F$ in the direction $\phi$. Polya shows that $k(\phi)=h(-\phi)$.

THEOREM 6.1. A function $f$ is in $G_{0}(\gamma)$ if and only if it is of the form

$$
f(\lambda)=\int_{-\infty}^{\infty} e^{-\lambda \xi} G(\xi) d \xi
$$

where

$$
G(\xi)= \begin{cases}F_{+}(\xi) & (0 \leq \xi<\infty), \\ F_{-}(\xi) & (-\infty<\xi<0),\end{cases}
$$

and $F_{+}$and $F_{-}$are entire functions of exponential type satisfying

$$
\begin{aligned}
& \left|F_{+}(\xi)\right|=O\left(e^{\xi \delta_{+}}\right), \quad \delta_{+}<-\gamma, \text { as } \xi \rightarrow+\infty, \\
& \left|F_{-}(\xi)\right|=O\left(e^{\xi \delta_{-}}\right), \quad \delta_{-}>\gamma, \text { as } \xi \rightarrow-\infty
\end{aligned}
$$

Proof. Let $f \in G_{0}(\gamma)$. Then we may write

$$
\begin{aligned}
f(\lambda) & =\frac{1}{2 \pi i} \int_{\Omega_{1}} \frac{f(\lambda)}{\xi-\lambda} d \xi+\frac{1}{2 \pi i} \int_{\Omega_{2}} \frac{f(\xi)}{\xi-\lambda} d \xi \\
& =f_{-}(\lambda)+f_{+}(\lambda),
\end{aligned}
$$

where $\Omega_{1}$ and $\Omega_{2}$ are bounded clockwise contours lying in $R(\lambda)>\gamma$ and $R(\lambda)<-\gamma$, respectively, which enclose the sets of singularities of $f$ which are in these two half-planes. The functions $f_{-}$and $f_{+}$are in $G_{0}(\gamma)$, and the decomposition is unique. Then

$$
f_{+}(\lambda)=\int_{0}^{\infty} e^{-\lambda \xi} F_{+}(\xi) d \xi, \quad R(\lambda)>c_{+},
$$

where $F_{+}(\xi)$ is entire of type $c_{+}$. Since the singularities of $f_{+}$lie in $R(\lambda)<-\gamma$,

$$
k(\pi)=h(0)<-\gamma
$$

and thus

$$
\left|F_{+}(\xi)\right|=O\left(e^{\xi \delta}+\right)
$$


where $\delta_{+}<-\gamma$ as $\xi \longrightarrow+\infty$, and the integral (6.3) converges absolutely for $R(\lambda)>-\gamma \cdot$

One shows that

$$
f_{-}(\lambda)=\int_{-\infty}^{0} e^{-\lambda \xi} F_{-}(\xi) d \xi
$$

where $-F_{-}(\xi)$ is the entire function associated with $f_{-}$by $(6.1)$ and $(6.2)$ by setting

$$
\lambda=-\mu, g(\mu)=f_{-}(\lambda)
$$

as the singularities of $g$ lie in $R(\mu)<-\gamma$.

Conversely, if

$$
f_{+}(\lambda)=\int_{0}^{\infty} e^{-\lambda \xi} F_{+}(\xi) d \xi,
$$

where $F_{+}$is an entire function of exponential type with the indicated order property, $f_{+}$is analytic at infinity and its singularities lie in $R(\lambda)<-\gamma$. The case of $f_{-}$is similar.

7. An inversion theorem. We now prove a result which, when $T$ is the operator of differentiation in spaces of functions on the real line, will yield the inversion of many common convolution transforms by differential operators of infinite order (see $(1.2)$ and $(8.3))$.

Let $f$ be in $U(\gamma)$ and $[f\{T\}]^{-1}$ exist. If $1 / f$ is in $\mathcal{L}(m, \gamma)$ for some $m$, the calculus shows that

$$
[f\{T\}]^{-1}=\left(f^{-1}\right)(T)
$$

When this is not the case, however, we can often construct the inverse as a pointwise limit of polynomials or other operators. The idea is to find a sequence $\left\{h_{n}\right\}$ of functions in $\mathcal{L}(\infty, \gamma)$ such that

$$
h_{n}(\lambda) f(\lambda) \longrightarrow 1
$$

suitably near $\sigma(T)$. The functions $h_{n}(\lambda) f(\lambda)$ may be treated by the calculus, and under proper conditions the sequence

$$
\left(h_{n} f\right)(T)=h_{n}(T) f(T)
$$


should converge strongly to $l$. We shall call a sequence $\left\{h_{n}\right\}$ an inverting sequence for $f \in U(\gamma)$ if

(1) $h_{n}(\lambda) f(\lambda) \in U(\gamma) n=1,2, \ldots$ with a common strip $[-r, r](r>\gamma)$ of absolute convergence;

(2) $\lim _{n \rightarrow \infty} h_{n}(\lambda) f(\lambda)=1 \quad(-r \leq \sigma \leq r)$;

(3) for some integer $k \geq 0$,

$$
\lim _{n \rightarrow \infty} \int_{\Gamma_{r}}\left|\lambda^{-k}\left[1-h_{n}(\lambda) f(\lambda)\right]\right||d \lambda|=0 .
$$

Note that uniform boundedness of $h_{n}(\lambda) f(\lambda)$ implies (3) if $k=2$.

Lemma 7.1. If $h \in \mathcal{L}(m, \gamma)$ and $f$ and $h f$ are in $V(\gamma)$, then

$$
(h f)\{T\}=\widetilde{h}(T) f\{T\} .
$$

Proof. Given $x$ in $X$ we pick $x_{i}(i=1,2, \ldots$,$) in D_{m+2}(T)$ converging to $x$. Then $f\{T\} x_{i}$ and $(h f)\{T\} x_{i}$ converge to $f\{T\} x_{i}$ and $(h f)\{T\} x_{i}$ respectively. Since for each $i$,

$$
(h f)\{T\} x_{i}=h(T) f\{T\} x_{i}=\tilde{h}(T) f\{T\} x_{i},
$$

and $\tilde{h}(T)$ is closed, the result follows.

THЕOREM 7.1. If $\left\{h_{n}\right\}$ is an inverting sequence for $f \in U(\gamma)$, then

$$
\lim _{n \rightarrow \infty} \widetilde{h_{n}}(T) f\{T\} x=x
$$

for each $x$ in $D_{k}(T)$. The limit holds for all $x$ in $X$ if and only if the transformations $\left(h_{n} f\right)\{T\}$ are uniformly bounded.

Proof. For each $n$,

$$
\widetilde{h}_{n}(T) f\{T\}=\left(h_{n} f\right)\{T\}
$$

by the lemma. Now if $x \in D_{k}(T)$ we may write

$$
\left(h_{n} f\right)(T) x=\sum_{i=0}^{k-1} \frac{\left(h_{n} f\right)^{(i)}(0) T^{i} x}{i !}+\frac{1}{2 \pi i} \int_{\Gamma_{r}} \frac{h_{n}(\lambda) f(\lambda) R_{\lambda}(T) T^{k} x}{\lambda^{k}} d \lambda .
$$

By condition (2), all terms in the summation go to zero except the first which 
converges to $x$. The last term by (3) converges to

$$
\frac{1}{2 \pi \mathrm{i}} \int_{\Gamma_{\boldsymbol{r}}} \frac{R_{\lambda}(T) T^{k} x}{\lambda^{k}} d \lambda .
$$

But this integral vanishes by the argument to establish (2.6). Since $D_{k}(T)$ is dense in $X$ we conclude the last statement from the Banach-Steinhaus theorem and the principle of uniform boundedness $[3$, p. 25].

In applications we shall take $h_{n}(\lambda)$ of the form $p_{n}(\lambda)$ or $e^{c_{n} \lambda} p_{n}(\lambda)$, where $p_{n}$ is a polynomial and $c_{n}$ is real. In each case,

$$
h_{n}(T)=\widetilde{h}_{n}(T)
$$

For the former this was proved at the end of $\S 4$. The latter case is left to the reader.

8. Examples. An important application of the theory of the preceding sections is found by taking for $T$ the operator of differentiation in certain spaces of complex-valued functions defined on the boundary of the unit circle or on the real line. Where functions in the spaces we consider are defined on the line, the spectrum of $T$ is the imaginary axis, whereas $\sigma(T)$ consists of the integral points of the imaginary axis when the functions in the space are defined on the circumference of the circle. For this reason we shall call these two groups of spaces the continuous and discrete cases respectively.

\section{Continuous:}

1. $C[-\infty, \infty]$,

$$
\begin{aligned}
& \|x\|=\sup _{t}|x(t)|, \\
& D(T)=\left(x \mid x^{\prime}(t) \in C[-\infty, \infty]\right) .
\end{aligned}
$$

2. $L_{p}(-\infty, \infty)(1 \leq p<\infty)$,

$$
\begin{aligned}
\|x\|= & \left(\int_{-\infty}^{\infty}|x(t)|^{p} d t\right)^{1 / p}, \\
D(T)= & (x \mid x(t) \text { is absolutely continuous on each finite interval and } \\
& \left.x^{\prime}(t) \in L_{p}(-\infty, \infty)\right) .
\end{aligned}
$$

Discrete:

1. $C[-\pi, \pi]$, 


$$
\begin{aligned}
& \|x\|=\sup _{t}|x(t)|, \\
& D(T)=\left(x \mid x^{\prime}(t) \in C[-\pi, \pi]\right) .
\end{aligned}
$$

2. $L_{p}(-\pi, \pi)(1 \leq p<\infty)$,

$$
\begin{aligned}
& \|x\|=\left(\int_{-\pi}^{\pi}|(t)|^{p} d t\right)^{1 / p}, \\
& D(T)=\left(x \mid x(t) \text { is absolutely continuous and } x^{\prime}(t) \in L_{p}(-\pi, \pi)\right) .
\end{aligned}
$$

In the discrete case the functions are, of course, periodic, and an $x$ in $C[-\infty, \propto]$ has limits at $+\infty$ and $-\infty$.

Well-known theorems show that $D(T)$ and, in fact, $D_{\infty}(T)$ are dense in all these spaces. One shows easily that $T$ is closed. It follows that $(\lambda I-T)$ is closed for any $\lambda$, and $(\lambda I-T)^{-1}$ is closed when it exists. Since a closed transformation defined on the whole space is bounded [3, p. $\left.30^{\circ}\right]$, the resolvent $R_{\lambda}(T)$ will exist if and only if $\lambda$ is such that the differential equation

$$
\lambda y(t)-y^{\prime}(t)=x(t)
$$

has a unique solution $y$ in $X$ for each $x$ in $X$. Then

$$
y=R_{\lambda}(T) x .
$$

One shows easily in the continuous case (compare with 5.3 ) that

$$
R_{\lambda}(T) x(t)= \begin{cases}\int_{0}^{\infty} e^{-\lambda \xi} x(t+\xi) d \xi & (R(\lambda)>0), \\ -\int_{-\infty}^{0} e^{-\lambda \xi} x(t+\xi) d \xi & (R(\lambda)<0),\end{cases}
$$

and

$$
\exists(\xi) x(t)=x(t+\xi)
$$

Also

$$
\left\|R_{\lambda}(T)\right\| \leq \frac{1}{|\sigma|}
$$

When $f \in G(y)$ (as $\gamma=0$ we shall write just $Q$ hereafter ), that is, 


$$
f(\lambda)=\int_{-\infty}^{\infty} e^{-\lambda \xi} G(\xi) d \xi
$$

then

$$
f(T) x(t)=\int_{-\infty}^{\infty} G(\xi) x(t-\xi) d \xi
$$

Also

$$
\|f(T)\| \leq \int_{-\infty}^{\infty}|G(\xi)| d \xi
$$

in all the spaces.

Now in the discrete case the formulas above for the continuous case are all valid if one interprets $x(t)=x(t+2 n \pi)$. However, with this identity, they may be given the more convenient form

$$
R_{\lambda}(T) x(t)=\left\{\begin{array}{lll}
\frac{1}{1-e^{-2 \pi \lambda}} \int_{0}^{2 \pi} e^{-\lambda \xi} y(t+\xi) d \xi & (R(\lambda)>0), \\
\frac{-1}{1-e^{2 \pi \lambda}} \int_{-2 \pi}^{0} e^{-\lambda \xi} y(t+\xi) d \xi & (R(\lambda)<0) .
\end{array}\right.
$$

Another representation is

$$
\begin{array}{r}
R_{\lambda}(T) x(t)=\frac{2 e^{\lambda t}}{\sinh \pi \lambda}\left[e^{-\lambda \pi} \int_{-\pi}^{t} e^{-\lambda \xi} x(\xi) d \xi+e^{\lambda \pi} \int_{t}^{\pi} e^{-\lambda \xi} x(\xi) d \xi\right] \\
(\lambda \neq i n, n=0, \pm 1, \pm 2, \cdots) .
\end{array}
$$

For $f \in G$,

$$
f(T) x(t)=\int_{-\pi}^{\pi} H(\xi) x(t-\xi) d \xi,
$$

where

$$
H(\xi)=\sum_{n=-\infty}^{\infty} G(\xi+2 n \pi) .
$$

If we use the Fourier representation 


$$
x(t) \sim \sum_{n=-\infty}^{\infty} x_{n} e^{i n t}
$$

then

$$
R_{\lambda}(T) x(t) \sim \sum_{n=-\infty}^{\infty} \frac{x_{n} e^{i n t}}{\lambda-i n}
$$

and, more generally,

$$
f(T) x(t) \sim \sum_{n=-\infty}^{\infty} f(\text { in }) x_{n} e^{i n t}
$$

where the numbers $f($ in $)$ are the Fourier coefficients of $H(\xi)$. Again,

$$
\left\|R_{\lambda}(T)\right\| \leq \frac{1}{|\sigma|}
$$

and

$$
\|f(T)\| \leq \int_{-\pi}^{\pi}|H(\xi)| d \xi=\int_{-\infty}^{\infty}|G(\xi)| d \xi
$$

When $p=2$, one may show further that

$$
\|f(T)\|=\sup _{\lambda \in \sigma(T)}|f(\lambda)|
$$

in both the discrete and continuous cases. This and the above facts are well known. The transformations $f(T)$ are special cases of factor transforms for which one may refer to $[3$, p. 344, 361 ]; see also [1, p.99].

In view of these remarks we may state a corollary of Theorem 7.1 in the following convenient form.

THEовем 8.1. Let

$$
f(\lambda)=\int_{-\infty}^{\infty} e^{-\lambda \xi} G(\xi) d \xi
$$




$$
h_{n}(\lambda)=e^{c_{n} \lambda} p_{n}(\lambda),
$$

where the numbers $c_{n}$ are real and the $p_{n}$ are polynomials. If $f(\lambda)$ and

$$
h_{n}(\lambda) f(\lambda)=\int_{-\infty}^{\infty} e^{-\lambda \xi} G_{n}(\xi) d \xi
$$

have a common strip $[-r, r], r>0$ of absolute convergence, and in this strip

$$
\left|h_{n}(\lambda) f(\lambda)\right| \leq M
$$

and

$$
\lim _{n \rightarrow \infty} h_{n}(\lambda) f(\lambda)=1,
$$

then:

(a) if $x \in L_{p}(-\infty, \infty)(p \geq 1)$, or $C[-\infty, \infty]$, and $x \in D_{2}(T)$,

$$
\lim _{n \rightarrow \infty} h_{n}(T) \int_{-\infty}^{\infty} G(\xi) x(t-\xi) d \xi=x(t)
$$

in norm. If for some $M^{\prime}$,

$$
\int_{-\infty}^{\infty}\left|G_{n}(\xi)\right| d \xi \leq M^{\prime} \quad(n=1,2, \ldots),
$$

then (8.1) holds for all $x$.

(b) If $x \in L_{2}(-\infty, \infty)$, the limit (8.1) holds for all $x$ without (8.2).

Actually the theorem is too restrictive in $L_{2}(-\infty, \infty)$. Since $-i T$ is selfadjoint in this space [12, p.441], we may use the calculus of Stone and von Neumann (see $\$ 4)$. With this calculus it is sufficient for the conclusion that $h_{n}(\lambda) f(\lambda)$ be defined and converge boundedly to unity on the imaginary axis.

As an application of the theorem above we obtain for function spaces an inversion theorem due to Hirschman and Widder [7]. Let

$$
f(\lambda)=[E(\lambda)]^{-1}, E(\lambda)=\prod_{k=1}^{\infty}\left(1-\frac{\lambda}{a_{k}}\right) e^{\lambda / b_{k}},
$$

where $a_{k}=b_{k}+i c_{k}(k=1,2, \ldots)$ is a sequence of complex numbers such that 


$$
\sum_{k=1}^{\infty}\left(\frac{1}{b_{k}}\right)^{2}<\infty, \quad \sum_{k=1}^{\infty}\left(\frac{c_{k}}{b_{k}}\right)^{2}<\infty
$$

If

$$
h_{n}(\lambda)=e^{d_{n} \lambda} \prod_{k=1}^{n}\left(1-\frac{\lambda}{a_{k}}\right) e^{\lambda / b_{k}} \quad(n=1,2, \cdots),
$$

where the $d_{n}$ are real numbers approaching zero, then the conditions of the theorem on the functions $f(\lambda), h_{n}(\lambda) f(\lambda)(n=1,2, \ldots)$ are satisfied in any closed vertical strip free of zeros of $E(\lambda)$. Letting

$$
\begin{gathered}
G(\xi)=\frac{1}{2 \pi i} \int_{-i \infty}^{i \infty} f(\lambda) e^{\lambda \xi} d \lambda, \\
G_{n}(\xi)=\frac{1}{2 \pi i} \int_{-i \infty}^{i \infty} h_{n}(\lambda) f(\lambda) e^{\lambda \xi} d \lambda
\end{gathered}
$$

these authors show that

$$
\int_{-\infty}^{\infty}\left|G_{n}(\xi)\right| d \xi \leq M<\infty
$$

Thus

(8.3) $\lim _{n \rightarrow \infty} \Im\left(d_{n}\right) \prod_{k=1}^{n}\left(1-\frac{T}{a_{k}}\right) \zeta\left(\frac{1}{b_{k}}\right) \int_{-\infty}^{\infty} G(\xi) x(t-\xi) d \xi=x(t) \quad\left(T=\frac{d}{d t}\right)$, in norm for $x$ in any of the spaces. We list below the kernels of some common transforms with their bilateral transforms which fall under this discussion:

$$
\begin{array}{l|c}
\text { Laplace } & e^{\xi} e^{-e \xi} \\
\text { Stieltjes } & \frac{1}{2 \pi} \operatorname{sech} \frac{\xi}{2} \\
\text { Meijer } \quad \frac{2}{\pi} \cos \frac{\pi \nu}{2} e^{\xi} K_{\nu}(\xi) & \frac{1}{\cos \pi \lambda} \\
\frac{\cos (\pi \nu / 2) \Gamma((1-\nu-\lambda) / 2) \Gamma((1+\nu-\lambda) / 2)}{\pi 2^{\lambda}} \quad\left(-\frac{1}{2} \leq \nu \leq \frac{1}{2}\right) .
\end{array}
$$


Hirschman and Widder have studied inversion formulas of this sort in great detail (see $[4,5,6,7,16]$; see also Pollard [10]). Their results involve the formal differential operator $D=d / d t$, and are concerned with inversion at particular points rather than in the norm topology of function spaces. Their proofs are quite different, involving a convergence argument with the kernels $G_{n}(\xi)$ in contrast to the present method of first proving inversion for a dense set of functions. On the other hand, the present method seems unsuitable for obtaining local results.

Similar inversion formulas have been proved for $L_{2}(-\infty, \infty)$ by $\mathrm{H}$. Pollard [9] by use of Fourier transform methods. He needs only to prove that the products $h_{n}(\lambda) f(\lambda)$ converge boundedly to unity on the axis. However, in each case he considers one can show this is true in a strip of positive width. In several cases we are unable to show (8.2), for example in the case of the Weierstrass transform where

$$
G(\xi)=\frac{e^{-\xi^{2}}}{\sqrt{\pi}}, f(\lambda)=e^{\lambda^{2} / 4}, \text { and } h_{n}(\lambda)=\left(1-\frac{\lambda^{2}}{4 n}\right)^{n} \text {, }
$$

or the partial sums in the series for $f(\lambda)$, and in the case of the Stieltjes and Laplace transforms when $h_{n}$ are the partial sums.

As a final application, we give an example for the case of the circle. Here the Weierstrass transform takes the form either of a transformation of series

$$
x(t) \sim \sum_{n=-\infty}^{\infty} x_{n} e^{i n t}, f(T) x(t)=\sum_{n=-\infty}^{\infty} x_{n} e^{i n t-n^{2} / 4},
$$

or a finite convolution

$$
f(T) x(t)=\frac{1}{2 \pi} \int_{-\pi}^{\pi} \theta_{3}(\xi, 1 / 4) x(t-\xi) d \xi
$$

where

$$
\begin{aligned}
\theta_{3}(\xi, \alpha) & =\sqrt{\pi / \alpha} \sum_{n=-\infty}^{\infty} e^{-(\xi+2 n \pi)^{2} / 4 \alpha} \\
& =1+2 \sum_{n=1}^{\infty} e^{-n^{2} \alpha} \cos n \xi
\end{aligned}
$$


is the theta function occurring in the theory of heat conduction [3, p. 402]. For any $x$ in $L_{2}(-\pi, \pi)$ or $x$ in $D_{2}(T)$ of the other spaces,

$$
\lim _{n \rightarrow \infty}\left(1-\frac{T^{2}}{4 n}\right)^{n} f(T) x(t)=x(t)
$$

in norm.

\section{REFERENCES}

1. S. Bochner and K. Chandrasekharan, Fourier transforms, Ann. of Math. Studies No. 19, Princeton, 1949.

2. N. Dunford, Spectral theory I. Convergence to projections, Trans. Amer. Math. Soc. 54 (1943), $185-217$.

3. E. Hille, Functional analysis and semi-groups. Amer. Math. Soc. Coll. Publications, vol. 31, New York, 1948.

4. I. I. Hirschman, Jr. and D. V. Widder, An inversion and representation theory for convolution transforms with totally positive kernels, Proc. Nat. Acad. Sci. U.S.A. 34 (1948), $152-156$.

5. Generalized inversion formulas for convolution transforms, Duke Math. J. 15 (1948), 659-696.

6. The inversion of a general class of convolution transforms, Trans. Amer. Math. Soc. 66 (1949), $135-201$.

7. $211-225$.

Convolution transforms with complex kernels, Pacific J. Math. 1(1951),

8. B. Nagy, Spektraldarstellung linearer Transformationen des Hilbertschen Raumes, Berlin, 1942.

9. H. Pollard, Integral transforms, Duke Math. J. 13 (1946), 307-330.

10. Integral transforms II. Ann. of Math. 49 (1948), 956-965.

11. G. Polya, Untersuchungen uber Lücken und Singularitäten von Potenzreihen Math. Z. 29 (1929), 549-640.

12. M. H. Stone, Linear transformations in Hilbert space and their applications to analysis, Amer. Math. Soc. Coll. Publications, vol. 15, New York, 1932.

13. A. E. Taylor, Analysis in complex Banach spaces, Bull. Amer. Math. Soc. 49 (1943), 652-669.

14. 189-224.

15. E. C. Titchmarsh, The theory of functions, 2nd ed., Oxford, 1939.

16. D. V. Widder, Inversion formulas for convolution transforms, Duke Math J. 14 (1947), 217 - 249.

17. The Laplace transform, Princeton, 1946. 


\section{PACIFIC JOURNAL OF MATHEMATICS}

\section{EDITORS}

\section{R. U. FOEINSON}

University of California

Berkeley 4, California

E. HewitT

University of Washington

Seattle 5 , Washington
P. P. DILWOR TH

California Institute of Technology

Pasadena 4, California

E. F. BECKENBACH

University of California

Los Angeles 24, California

\section{ASSOCIATE EDITORS}

$\begin{array}{llll}\text { H. BUSEMANN } & \text { P. R. HALMOS } & \text { BØRGE JESSEN } & \text { J. J. STOKER } \\ \text { HERBERT FFDERER } & \text { IIEINZ HOPF } & \text { PAUL LÉVY } & \text { E. G. STRAUS } \\ \text { MARSHALL, IIALI } & \text { R. D. JAMES } & \text { GEORGE PÓLYA } & \text { KÖSAKU YOSIDA }\end{array}$

\section{SPONSORS}

UNIVERSITY OF BRITISH COLUMBIA

CALIFORNIA INSTITUTE OF TECHNOLOGY.

UNIVERSITY OF CAIJIFORNIA, BERKELEY

UNIVERSITY OF CAIJIFORNIA, DAVIS

UNIVERSITY OF CALIFORNIA, LOS ANGELES

UNIVERSITY OF CALIFORNI A, SANTA BARBARA

UNIVERSITY OF NEVADA

OREGON STATE COLLEGE

UNIVERSITY OF OREGON

\author{
UNIVERSITY OF SOU TIERN CALIFORNIA \\ STANFORD RESEARCH INSTITUTE \\ STANFORD UNIVERSITY \\ WASHINGTON STATE COLLEGE \\ UNIVERSITY OF WASHINGTON \\ AMERICAN MATHEMATICAL SOCIETY \\ NATIONAL BUREAU OF STANDARDS, \\ INSTITUTE FOR NUMERICAL ANALYSIS
}

Mathematical papers intended for publication in the Pacific Journal of Mathematics should be typewritten (double spaced), and the author should keep a complete copy. Manuscripts may be sent to any of the editors except Robinson, whose term expires with the completion of the present volume; they might also be sent to M.M. Schiffer, Stanford University, Stanford, California, who is succeeding Robinson. All other communications to the editors should be addressed to the managing editor, E. F. Beckenbach, at the address given above.

Authors are entitled to receive 100 free reprints of their published papers and may obtain additional copies at cost.

The Pacific Journal of Mathematics is published quarterly, in March, June, September, and December. The price per volume (4 numbers) is $\$ 8.00$; single issues, $\$ 2.50$. Special price to individual faculty members of supporting institutions and to individual members of the American Mathematical Society: $\$ 4.00$ per volume; single issues, $\$ 1.25$.

Subscriptions, orders for back numbers, and changes of address should be sent to the publishers, University of California Press, Berkeley 4, California.

Printed at Ann Arbor, Michigan. Entered as second class matter at the Post Office, Berkeley, California.

\section{UNIVERSITY OF CALIFORNIA PRESS • BERKELEY AND LOS ANGELES}




\section{Pacific Journal of Mathematics}

\section{Vol. 3, No. 2 \\ April, 1953}

William George Bade, An operational calculus for operators with spectrum

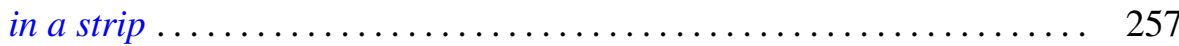

E. F. Beckenbach and Lloyd Kenneth Jackson, Subfunctions of several variables ..................................... 291

David Blackwell, Extension of a renewal theorem ................. 315

L. Carlitz, Some theorems on the Schur derivative ................ 321

Paul Arnold Clement, Generalized convexity and surfaces of negative curvature..................................... 333

Merrill M. Flood, On the Hitchcock distribution problem ............... 369

Watson Bryan Fulks, On the unique determination of solutions of the heat

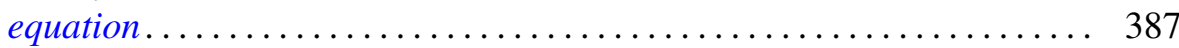

John W. Green, Length and area of a convex curve under affine transformation .................................... 393

William Gustin, An isoperimetric minimax .................. 403

Arthur Eugene Livingston, Some Hausdorff means which exhibit the Gibbs'

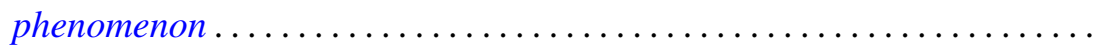

Charles Loewner, On generation of solutions of the biharmonic equation in the plane by conformal mappings ..................... 417

Gábor Szegő, Remark on the preceding paper of Charles Loewner ....... 437

Imanuel Marx and G. Piranian, Lipschitz functions of continuous

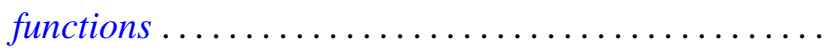

Ting-Kwan Pan, The spherical curvature of a hypersurface in Euclidean space ..

Ruth Lind Potter, On self-adjoint differential equations of second order ...

E. H. Rothe, A note on the Banach spaces of Calkin and Morrey...

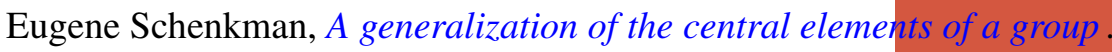

A. Seidenberg, A note on the dimension theory of rings .. . . 